\title{
Hadronic scattering of charmed mesons
}

\author{
Ziwei Lin, C. M. Ko, and Bin Zhang \\ Cyclotron Institute and Physics Department, Texas A\&M University, College Station, Texas 77843
}

(Received 4 May 1999; published 4 January 2000)

\begin{abstract}
The scattering cross sections of charm mesons with hadrons such as the pion, rho meson, and nucleon are studied in an effective Lagrangian. In heavy ion collisions, rescattering of produced charm mesons by hadrons affects the invariant mass spectra of both charm meson pairs and dileptons resulting from their decays. These effects are estimated for heavy ion collisions at Super Proton Synchrotron energies and are found to be significant.
\end{abstract}

PACS number(s): 25.75.-q, 13.75.Lb, 14.40.Lb

\section{INTRODUCTION}

Recently, experiments on heavy ion collisions at CERN Super Proton Synchrotron (SPS) by the HELIOS-3 [1] and NA50 [2] Collaborations have shown an enhanced production of dileptons of intermediate masses $(1.5<M<2.5$ $\mathrm{GeV}$ ). In one explanation, this enhancement is attributed to dilepton production from secondary meson-meson interactions [3], while in another it was proposed that dileptons from charm meson decays could also contribute appreciably in this invariant mass region [4]. In the latter case, one of the present authors has shown, based on a schematic model, that if one assumes that the transverse mass spectra of charm mesons become hardened as a result of final state rescattering with hadrons, the invariant mass spectrum of dimuons from decays of charm mesons would also become hardened, and more dimuons would then have an invariant mass between 1.5 and $2.5 \mathrm{GeV}$. Although charm quark production from hadronic interactions has been extensively studied using perturbative QCD $[5,6]$, not much has been done in studying charm meson interactions with hadrons. Knowledge of charm meson interactions with hadrons is important, as whether charm mesons develop a transverse flow depends on how strongly they interact with other hadrons as they propagate through the matter.

In this paper, we shall first introduce in Sec. II an effec- tive Lagrangian to describe the interactions of charm mesons with pion, rho, and nucleon. Using the coupling constants and cutoff parameters at the vertices determined either empirically or from symmetry arguments, we evaluate the scattering cross section of charm mesons with hadrons. Effects of hadronic scattering on the charm meson transverse momentum spectrum and the dimuon invariant mass spectrum from charm meson decays are then estimated in Sec. III based on a schematic model for the time evolution of heavy ion collision dynamics. In Sec. IV, we summarize our results and discuss the uncertainties involved in the studies.

\section{CHARM MESON INTERACTIONS WITH HADRONS}

\section{A. Lagrangian}

We consider the scattering of charm mesons $\left(D^{+}, D^{-}\right.$, $D^{0}, \bar{D}^{0}, D^{*+}, D^{*-}, D^{* 0}$, and $\bar{D}^{* 0}$ ) with pion, rho, and nucleon. If SU(4) symmetry were exact, interactions between pseudoscalar and vector mesons could be described by the Lagrangian

$$
\mathcal{L}_{\mathrm{PPV}}=i g \operatorname{Tr}\left(P^{\dagger} V^{\mu \dagger} \partial_{\mu} P\right)+\text { H.c. }
$$

where $P$ and $V$ represent, respectively, the $4 \times 4$ pseudoscalar and vector meson matrices

$$
P=\frac{1}{\sqrt{2}}\left(\begin{array}{cccc}
\frac{\pi^{0}}{\sqrt{2}}+\frac{\eta}{\sqrt{6}}+\frac{\eta_{c}}{\sqrt{12}} & \pi^{+} & K^{+} & \bar{D}^{0} \\
\pi^{-} & -\frac{\pi^{0}}{\sqrt{2}}+\frac{\eta}{\sqrt{6}}+\frac{\eta_{c}}{\sqrt{12}} & K^{0} & D^{-} \\
K^{-} & \bar{K}^{0} & -\sqrt{\frac{2}{3}} \eta+\frac{\eta_{c}}{\sqrt{12}} & D_{s}^{-} \\
D^{0} & D^{+} & D_{s}^{+} & -\frac{3 \eta_{c}}{\sqrt{12}}
\end{array}\right),
$$




$$
V=\frac{1}{\sqrt{2}}\left(\begin{array}{cccc}
\frac{\rho^{0}}{\sqrt{2}}+\frac{\omega}{\sqrt{6}}+\frac{J / \psi}{\sqrt{12}} & \rho^{+} & K^{*+} & \bar{D}^{* 0} \\
\rho^{-} & -\frac{\rho^{0}}{\sqrt{2}}+\frac{\omega}{\sqrt{6}}+\frac{J / \psi}{\sqrt{12}} & K^{* 0} & D^{*^{-}} \\
K^{*-} & \bar{K}^{* 0} & -\sqrt{\frac{2}{3} \omega+\frac{J / \psi}{\sqrt{12}}} & D_{s}^{*-} \\
D^{* 0} & D^{*+} & D_{s}^{*+} & -\frac{3 J / \psi}{\sqrt{12}}
\end{array}\right) .
$$

The above interaction Lagrangian may be considered as being motivated by the hidden gauge theory, in which there are no four-point vertices that involve two pseudoscalar mesons and two vector mesons. This is in contrast to the approach of using the minimal substitution to introduce vector mesons as gauge particles, where such four-point vertices do appear. It is, however, known that the two methods are consistent if one also includes in the latter approach the axial vector mesons, which are unfortunately not known for charm hadrons. Furthermore, gauge invariance in the latter approach cannot be consistently maintained if one uses the experimental vector meson masses, empirical meson coupling constants and form factors at interacting vertices. Expanding the Lagrangian in Eq. (1) in terms of the meson fields explicitly, we obtain the following Lagrangians for mesonmeson interactions:

$$
\begin{aligned}
& \mathcal{L}_{\pi D D^{*}}=i g_{\pi D D^{*}} D^{* \mu} \vec{\tau} \cdot\left[\bar{D}\left(\partial_{\mu} \vec{\pi}\right)-\left(\partial_{\mu} \bar{D}\right) \vec{\pi}\right]+\text { H.c. }, \\
& \mathcal{L}_{\rho D D}=i g_{\rho D D}\left[D \vec{\tau}\left(\partial_{\mu} \bar{D}\right)-\left(\partial_{\mu} D\right) \vec{\tau} \bar{D}\right] \cdot \vec{\rho}^{\mu}, \\
& \mathcal{L}_{\rho \pi \pi}=g_{\rho \pi \pi} \vec{\rho}^{\mu} \cdot\left(\vec{\pi} \times \partial_{\mu} \vec{\pi}\right),
\end{aligned}
$$

where the coupling constants $g_{\pi D D^{*}}, g_{\rho D D}$, and $g_{\rho \pi \pi}$ are related to the coupling constant $g$ via the $\mathrm{SU}(4)$ symmetry as shown below in Eq. (4).

In SU(3), the Lagrangian for meson-baryon interactions can be similarly written using the meson and baryon matri-

(a)

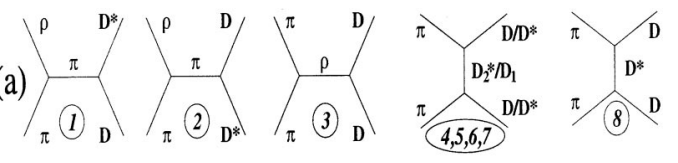

(b)

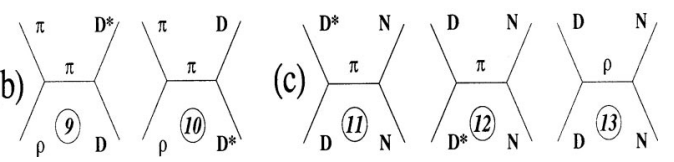

FIG. 1. Feynman diagrams for (a) $D \pi$, (b) $D \rho$, and (c) $D N$ scatterings. ces. The formulation becomes, however, more complicated in SU(4) where a more general tensor method is required [7]. The interaction Lagrangians needed for our study then include the following:

$$
\begin{gathered}
\mathcal{L}_{\pi N N}=-i g_{\pi N N} \bar{N} \gamma_{5} \vec{\tau} N \cdot \vec{\pi}, \\
\mathcal{L}_{D N \Lambda_{c}}=i g_{D N \Lambda_{c}}\left(\bar{N} \gamma_{5} \Lambda_{c} \bar{D}+\bar{\Lambda}_{c} \gamma_{5} N D\right) .
\end{gathered}
$$

In the above we have used the following conventions:

$$
\pi^{ \pm}=\frac{\pi_{1} \mp i \pi_{2}}{\sqrt{2}}, N=(p, n) \text { and } D=\left(D^{0}, D^{+}\right) .
$$

Again, SU(4) symmetry would relate the above coupling constants to each other with the introduction of one more parameter, as shown below in Eq. (8), because there are two $\mathrm{SU}(4)$-invariant Lagrangians for pseudoscalar meson and baryon interactions. We also need the following phenomenological Lagrangian:

$$
\mathcal{L}_{\rho N N}=g_{\rho N N} \bar{N}\left(\gamma^{\mu} \vec{\tau} \cdot \vec{\rho}_{\mu}+\frac{\kappa_{\rho}}{2 m_{N}} \sigma^{\mu \nu} \vec{\tau} \cdot \partial_{\mu} \vec{\rho}_{\nu}\right) N,
$$

where values of the coupling constants $g_{\rho N N}$ and $\kappa_{\rho}$ are well known as discussed below.

\section{B. Cross sections}

In Fig. 1, Feynman diagrams are shown for charm meson interactions with the pion (diagrams 1 to 8 ), the rho meson (diagrams 9 and 10), and the nucleon (diagrams 11 to 13). Explicit isospin states are not indicated. The spin and isospin-averaged differential cross sections for the $t$ channel and $u$ channel processes can be straightforwardly evaluated, and they are given by 


$$
\begin{aligned}
& \frac{d \sigma_{1}}{d t}=\frac{g_{\rho \pi \pi}^{2} g_{\pi D D^{*}}^{2}}{32 \pi s p_{i}^{2}} \frac{\left[m_{\rho}^{2}-2 m_{\pi}^{2}-2 t+\left(t-m_{\pi}^{2}\right)^{2} / m_{\rho}^{2}\right]\left[m_{D^{*}}^{2}-2 m_{D}^{2}-2 t+\left(t-m_{D}^{2}\right)^{2} / m_{D^{*}}^{2}\right]}{\left(t-m_{\pi}^{2}\right)^{2}}, \\
& \frac{d \sigma_{2}}{d t}=\frac{1}{3} \frac{d \sigma_{1}}{d t}, \\
& \frac{d \sigma_{3}}{d t}=\frac{g_{\rho \pi \pi}^{2} g_{\rho D D}^{2}}{32 \pi s p_{i}^{2}} \frac{\left(2 s+t-2 m_{\pi}^{2}-2 m_{D}^{2}\right)^{2}}{\left(t-m_{\rho}^{2}\right)^{2}} \\
& \frac{d \sigma_{9}}{d t}=\frac{1}{3} \frac{d \sigma_{1}}{d t}, \\
& \frac{d \sigma_{10}}{d t}=\frac{1}{9} \frac{d \sigma_{1}}{d t}, \\
& \frac{d \sigma_{11}}{d t}=\frac{3 g_{\pi D D^{*}}^{2} g_{\pi N N}^{2}}{64 \pi s p_{i}^{2}} \frac{(-t)\left[m_{D^{*}}^{2}-2 m_{D}^{2}-2 t+\left(t-m_{D}^{2}\right)^{2} / m_{D^{*}}^{2}\right]}{\left(t-m_{\pi}^{2}\right)^{2}} \\
& \frac{d \sigma_{12}}{d t}=\frac{1}{3} \frac{d \sigma_{11}}{d t}, \\
& \frac{d \sigma_{13}}{d t}=\frac{3 g_{\rho D D}^{2} g_{\rho N N}^{2}}{32 \pi s p_{i}^{2}} \frac{2\left(1+\kappa_{\rho}\right)^{2}\left[-s u+m_{N}^{2}(s+u)+m_{D}^{4}-m_{N}^{4}\right]-(s-u)^{2} \kappa_{\rho}\left(1+\kappa_{\rho} / 2+t \kappa_{\rho} / 8 m_{N}^{2}\right)}{\left(t-m_{\rho}^{2}\right)^{2}},
\end{aligned}
$$

where $p_{i}$ denotes the initial momentum of the two scattering particles in their center-of-mass frame.

For $s$-channel processes through charm meson resonances, shown by diagrams 4 to 8 , the cross section is taken to have a Breit-Wigner form

$$
\sigma=\frac{(2 J+1)}{\left(2 s_{1}+1\right)\left(2 s_{2}+1\right)} \frac{4 \pi}{p_{i}^{2}} \frac{\Gamma_{\mathrm{tot}}^{2} B_{\mathrm{in}} B_{\mathrm{out}}}{\left(s-M_{R}^{2}\right)^{2} / s+\Gamma_{\mathrm{tot}}^{2}},
$$

where $\Gamma_{\text {tot }}$ is the total width of the resonance, $B_{\text {in }}$ and $B_{\text {out }}$ are their decay branching ratios to the initial and final states, respectively. We note that diagrams 4 to 7 correspond to processes through the $D_{2}^{*}$ and $D_{1}$ resonances $D \pi \rightarrow D \pi$, $D \pi \rightarrow D^{*} \pi, \quad D^{*} \pi \rightarrow D \pi$ and $D^{*} \pi \rightarrow D^{*} \pi$, respectively, while diagram 8 represents the process $D \pi \rightarrow D \pi$ through the $D^{*}$ resonance. Total widths for $D_{2}^{*}$ and $D_{1}$ resonances are known, and they are $\Gamma_{D_{2}^{* 0}}=23 \mathrm{MeV}, \Gamma_{D_{2}^{*+}}=25 \mathrm{MeV}$, $\Gamma_{D_{1}^{0}}=18.9 \mathrm{MeV}$, and $\Gamma_{D_{1}^{+}}=28 \mathrm{MeV}$ [8]. For the width of $D^{*}$, only an upper limit is known, i.e., $\Gamma_{D^{* 0}}<2.1 \mathrm{MeV}$ and $\Gamma_{D^{*}+}<0.131 \mathrm{MeV}$. Studies based on the relativistic potential model [9] suggest that $\Gamma_{D^{* 0}} \simeq 42 \mathrm{KeV}$ and $\Gamma_{D^{*+}} \simeq 46$ $\mathrm{KeV}$, and we use these values in this paper. The branching ratios (BR) are known for $D^{*+}$ and $D^{* 0}$ [8], but not for $D_{2}^{*}$ and $D_{1}$. Experimental data show that for both $D_{2}^{* 0}$ and $D_{2}^{*+}$ decays one has $\Gamma\left(D \pi_{c h}\right) / \Gamma\left(D^{*} \pi_{c h}\right) \sim 2$. Since $D_{1}$ decays to $D^{*} \pi$ instead to $D \pi$ due to parity conservation, we assume
$\mathrm{B}\left(D_{1} \rightarrow D^{*} \pi\right)=1, \mathrm{~B}\left(D_{2}^{*} \rightarrow D^{*} \pi\right)=1 / 3$, and $\mathrm{B}\left(D_{2}^{*} \rightarrow D \pi\right)$ $=2 / 3$, neglecting possible decays of $D_{1}$ and $D_{2}^{*}$ to $D \rho$ and $D^{*} \rho$, respectively [10].

\section{Coupling constants}

For coupling constants, we use the empirical values $g_{\rho \pi \pi}=6.1$ [11], $g_{\pi D D *}=4.4, g_{\rho D D}=2.8[12], g_{\pi N N}=13.5$ [13], $g_{\rho N N}=3.25$, and $\kappa_{\rho}=6.1$ [14]. From SU(4) symmetry, as assumed in the Lagrangian in Eq. (1), one would expect the following relations among these couplings constants:

$$
\begin{aligned}
g_{\pi K K^{*}}(3.3) & =g_{\pi D D} *(4.4)=g_{\rho K K}(3.0)=g_{\rho D D}(2.8) \\
& =\frac{g_{\rho \pi \pi}}{2}(3.0) .
\end{aligned}
$$

One sees that the empirical values given in parentheses agree reasonably well with the prediction from SU(4) symmetry. Signs of the coupling constants are not specified as the possible interferences among diagrams 3,4 , and 8 are not included. We note that the coupling constant $g_{\pi D D} *$ is consistent with that determined from the $D^{*}$ width

$$
\Gamma_{D * \rightarrow \pi D}=\frac{g_{\pi D D^{*}}^{2} p_{f}^{3}}{2 \pi m_{D *}^{2}},
$$

where $p_{f}$ is the momentum of final particles in the $D^{*}$ rest frame. 


\section{Form factors}

To take into account the structure of hadrons, we introduce form factors at the vertices. For $t$-channel vertices, monopole form factors are used, i.e.,

$$
f(t)=\frac{\Lambda^{2}-m_{\alpha}^{2}}{\Lambda^{2}-t}
$$

where $\Lambda$ is a cutoff parameter, and $m_{\alpha}$ is the mass of the exchanged meson. For cutoff parameters, we use the empirical values $\Lambda_{\rho \pi \pi}=1.6 \mathrm{GeV}[11], \Lambda_{\pi N N}=1.3 \mathrm{GeV}$, and $\Lambda_{\rho N N}=1.4 \mathrm{GeV}$ [13]. However, there are no experimental information on $\Lambda_{\pi D D^{*}}$ and $\Lambda_{\rho D D}$, and their values are assumed to be similar to those determined empirically for strange mesons, i.e., $\Lambda_{\pi D D *}=\Lambda_{\pi K K^{*}}=1.8 \mathrm{GeV}, \Lambda_{\rho D D}$ $=\Lambda_{\rho K K}=1.8 \mathrm{GeV}$ [11]. For $s$-channel processes, shown in diagrams 4 to 8 , that are described by Breit-Wigner formula, no form factors are introduced.

\section{E. On-shell divergence}

The cross sections in Eq. (3) for diagrams 2 and 9 $\left(D \rho \leftrightarrow D^{*} \pi\right)$ are singular because the exchanged mesons can be on shell. Since the on-shell process describes a twostep process, their contribution needs to be subtracted from the cross section. This can be achieved by taking into account the medium effects which add an imaginary selfenergy to the mass of the exchanged pion as in Ref. [15]. We take the imaginary pion self-energy to be $50 \mathrm{MeV}$ and have checked that the calculated thermal average of the cross sections do not change much with values between 5 and 500 $\mathrm{MeV}$. We note that there are other ways to regulate this singularity [16].

\section{F. Thermal average}

We are interested in the thermal averaged cross sections for the processes shown in Fig. 1. For a process $1+2 \rightarrow 3$ +4 , where the initial-state particles 1 and 2 are both described by thermal distributions at temperature $T$, the thermal averaged cross section is given by

$$
\langle\sigma \mathrm{v}\rangle=\frac{\int_{z_{0}}^{\infty} d z\left[z^{2}-\left(\alpha_{1}+\alpha_{2}\right)^{2}\right]\left[z^{2}-\left(\alpha_{1}-\alpha_{2}\right)^{2}\right] K_{1}(z) \sigma\left(s=z^{2} T^{2}\right)}{4\left(1+\delta_{12}\right) \alpha_{1} K_{2}\left(\alpha_{1}\right) \alpha_{2} K_{2}\left(\alpha_{2}\right)} .
$$

In the above, $\alpha_{i}=m_{i} / T$ ( $i=1$ to 4$), z_{0}=\max \left(\alpha_{1}+\alpha_{2}, \alpha_{3}\right.$ $\left.+\alpha_{4}\right), \delta_{12}$ is 1 for identical initial-state particles and 0 otherwise, and $v$ is their relative velocity in the collinear frame, i.e.,

$$
\mathbf{v}=\frac{\sqrt{\left(k_{1} \cdot k_{2}\right)^{2}-m_{1}^{2} m_{2}^{2}}}{E_{1} E_{2}}
$$

In Fig. 2(a), we show the results for the thermal averaged cross sections as functions of temperature. It is seen that dominant contributions are from $D$ and $D^{*}$ scatterings by nucleon, $D$ scattering by pion via rho exchange, and $D$ scattering by rho meson via pion exchange. In obtaining these result, the rho meson mass is taken at its peak value of 770 $\mathrm{MeV}$.

\section{ESTIMATES OF RESCATTERING EFFECTS}

As shown in the schematic model of Ref. [4], if one assumes that charm mesons interact strongly in the final state hadronic matter, then their transverse mass $\left(m_{\perp}\right)$ spectra and pair invariant mass spectra would become harder than the initial ones as a result of the appreciable transverse flow of the hadronic matter. Dilepton decays of charm mesons would then lead to an enhanced yield of intermediate-mass dileptons in heavy ion collisions. In this section, we estimate the effects of hadronic rescattering on charm meson $m_{\perp}$ spectra and the invariant-mass distribution of dileptons from their decays in heavy ion collisions at SPS energies.
To characterize the scattering effects on charm mesons, we first determine the squared momentum transfer to a charm meson when it undergoes a scattering process $D_{1} X_{1}$ $\rightarrow D_{2} X_{2}$. In the rest frame of $D_{1}$, the squared momentum of the final charm meson $D_{2}$ is given by

$$
p_{0}^{2}=\frac{\left[\left(m_{D_{1}}+m_{D_{2}}\right)^{2}-t\right]\left[\left(m_{D_{1}}-m_{D_{2}}\right)^{2}-t\right]}{\left(2 m_{D_{1}}\right)^{2}}
$$

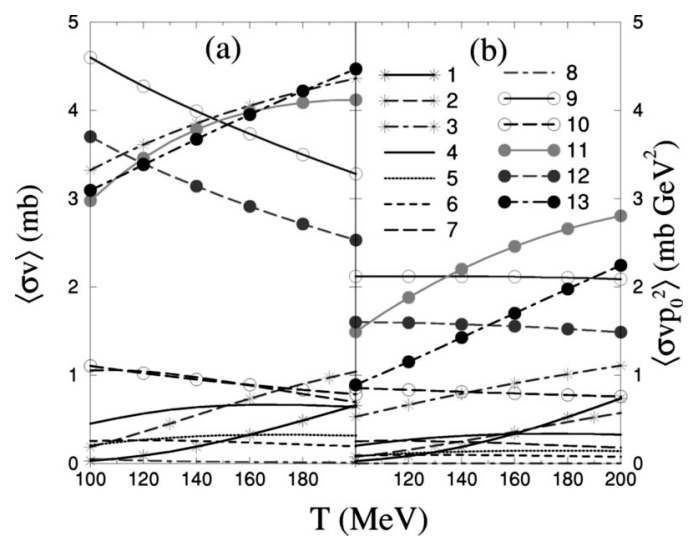

FIG. 2. Thermal average (a) $\langle\sigma \mathrm{v}\rangle$ and (b) $\left\langle\sigma \vee p_{0}^{2}\right\rangle$ of charm meson scattering cross sections as functions of temperature. Numbers labeling the curves correspond to the diagram numbers in Fig. 1. 
for $t$-channel processes with four momentum transfer $t$. For $u$ channel processes, one replaces $u$ for $t$ in the above expression.

We determine the total number of collisions suffered by a charm meson from its scattering cross sections and the time evolution of the hadron densities. In the charm meson local frame, we assume that the density evolution of hadrons is inversely proportional to the proper time, i.e.,

$$
\rho(\tau) \propto \frac{1}{\tau} .
$$

Neglecting the effect of transverse expansion on the density evolution, the total number of scatterings for a charm meson is then

$$
\begin{aligned}
N & =\int_{\tau_{0}}^{\tau_{F}} \sigma \mathrm{V} \rho d \tau=\sigma \mathrm{V} \rho_{0} \tau_{0} \ln \left(\frac{\tau_{F}}{\tau_{0}}\right) \\
& =\sigma \mathrm{\vee} \rho_{0} \tau_{0} \ln \left(\frac{t_{F}}{\tau_{0} \cosh y}\right) \simeq \sigma \mathrm{\vee} \rho_{0} \tau_{0} \ln \left(\frac{R_{\perp} m_{\perp}}{\tau_{0} p_{\perp}}\right)
\end{aligned}
$$

which leads to the following thermal average of the squared total momentum transfer due to scatterings:

$$
\left\langle p_{\mathrm{S}}^{2}\right\rangle=\left\langle N p_{0}^{2}\right\rangle=\left[\sum_{i=\pi, \rho, N, \ldots}\left\langle\sigma \mathrm{\vee} p_{0}^{2}\right\rangle_{i} \rho_{i 0}\right] \tau_{0} \ln \left(\frac{R_{\perp} m_{\perp}}{\tau_{0} p_{\perp}}\right) .
$$

In obtaining the above result, we have assumed the same initial and final proper times for the time evolution of different particle species that are involved in the scattering. Equation (6) shows that the relevant quantity is $\left\langle\sigma \vee p_{0}^{2}\right\rangle$ instead of the usual $\langle\sigma \mathrm{v}\rangle$. We show in Fig. 2(b) this thermal average for all scattering channels considered in the present study. It is seen that the dominant contributions to $\langle\sigma \mathrm{V}\rangle$ remain important for $\left\langle\sigma \vee p_{0}^{2}\right\rangle$ and the process involving $D^{*}$ scattering by rho meson via pion exchange also becomes significant.

Summing up contributions from the scattering channels in Figs. 1(a), 1(b), and 1(c) separately, and simply dividing by 2 to account for the average over $D$ and $D^{*}$, we obtain, at $T=150 \mathrm{MeV}$,

$$
\left\langle\sigma \vee p_{0}^{2}\right\rangle \simeq 1.1,1.5, \text { and } 2.7 \mathrm{mb} \mathrm{GeV}^{2}
$$

for $\pi, \rho$, and $N$ scatterings with charm mesons, respectively.

For central $\mathrm{Pb}+\mathrm{Pb}$ collisions at SPS energies, the initial particle numbers can be obtained from Ref. [17], i.e., there are $500 \pi, 220 \rho, 100 \omega, 80 \eta, 180 \mathrm{~N}, 60 \Delta$, and 130 higher baryon resonances. The initial densities at central rapidity can then be estimated using $\rho_{0} \tau_{0} \simeq(d N / d y) /\left(\pi R_{A}^{2}\right)$ $\simeq N /\left(4 \pi R_{A}^{2}\right)$. For a conservative estimate on the scattering effect, we only include $\pi, \rho$ and nucleon. The initial densities for pion, rho meson and nucleon are thus

$$
\rho_{0} \tau_{0} \simeq 0.79, \quad 0.35, \text { and } 0.28 \mathrm{fm}^{-2},
$$

respectively. Equation (6) then gives

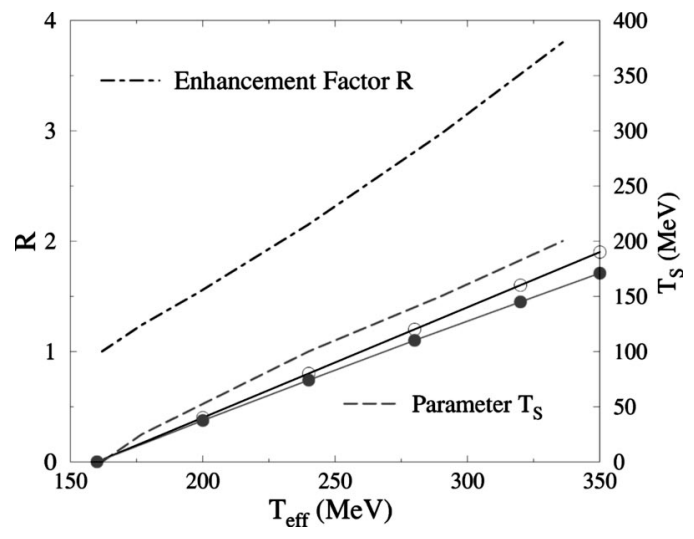

FIG. 3. The dimuon enhancement factor $R$ within the simulated NA50 acceptance and the equivalent temperature parameter $T_{S}$ due to scatterings as functions of the final inverse slope of charm mesons $T_{\text {eff }}$ [4]. The curve with open circles is from Eq. (A4), while the curve with filled circles is from Eq. (A6).

$$
\begin{aligned}
\left\langle p_{\mathrm{S}}^{2}\right\rangle \simeq & {\left[\left\langle\sigma \vee p_{0}^{2}\right\rangle_{\pi} \rho_{\pi 0}+\left\langle\sigma \vee p_{0}^{2}\right\rangle_{\rho} \rho_{\rho 0}\right.} \\
& \left.+\left\langle\sigma \vee p_{0}^{2}\right\rangle_{N} \rho_{N 0}\right] \tau_{0} \ln \left(\frac{R_{\perp}\left\langle m_{\perp}\right\rangle}{\tau_{0}\left\langle p_{\perp}\right\rangle}\right) \\
\simeq & (1.1 \times 0.79+1.5 \times 0.35+2.7 \times 0.28) / 10 \times \ln 16.7 \\
\simeq & 0.61\left(\mathrm{GeV}^{2}\right)
\end{aligned}
$$

In the above, we have taken $\tau_{0}=1$ fm and $R_{\perp} \simeq R_{A}$ $\simeq 1.2 A^{1 / 3} \mathrm{fm}$. We have also used the relations $\left\langle p_{\perp}\right\rangle$ $\sim \sqrt{\left\langle p_{\perp}^{2}\right\rangle} \simeq \sqrt{2 m T_{\text {eff }}}$ and $\left\langle m_{\perp}\right\rangle \simeq m+T_{\text {eff }}$ as given by Eq. (A2) in the Appendix. Since the charm meson $T_{\text {eff }}$ increases as a result of the rescatterings, $\left\langle p_{\mathrm{S}}^{2}\right\rangle$ needs to be determined self-consistently. However, because of the logarithmic dependence shown in Eq. (7), $\left\langle p_{\mathrm{S}}^{2}\right\rangle$ is not very sensitive to the value of $T_{\text {eff }}$, and we have taken $T_{\text {eff }}=200 \mathrm{MeV}$ in obtaining the above numerical results. We note that even though pions appear to be less important in Fig. 2, their contribution to the rescattering effect is important due to their high densities, as evident from the numerical values shown in Eq. (7).

The total squared momentum transfer from hadronic scatterings as given by Eq. (6) can be characterized by a temperature parameter $T_{\mathrm{S}}$, defined by Eq. (A3) in the Appendix. Using the values given in Eq. (7), we obtain $T_{\mathrm{S}} \simeq 96 \mathrm{MeV}$ from Eq. (A5) of the Appendix. From Fig. 3, which relates $T_{\mathrm{S}}$ to $T_{\text {eff }}$ and to the enhancement factor $R$ for dimuons from charm meson decays into the NA50 acceptance, this gives an effective inverse slope parameter of $T_{\text {eff }}=235 \mathrm{MeV}$ for the final charm meson $m_{\perp}$ spectrum if the initial one is taken to be $160 \mathrm{MeV}$, and a dimuon enhancement factor of about 2.1 is obtained.

\section{SUMMARY AND DISCUSSIONS}

In summary, we have calculated the cross sections for scatterings between charm mesons and hadrons such as pion, rho meson, and nucleon. Hadronic scatterings of charm mesons in heavy ion collisions can significantly affect the charm meson spectra and the dilepton spectra from charm 

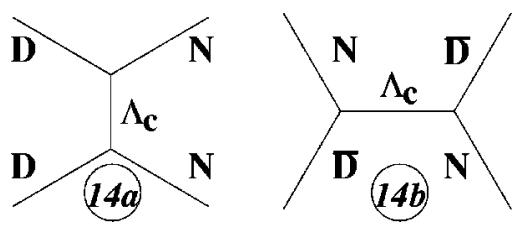

FIG. 4. Feynman diagrams for charm meson scattering with nucleon via the $\Lambda_{c}$ exchange.

meson decays. An estimate of this effect in heavy ion collisions at SPS energies is given, and we find that it leads to a hardened charm meson spectra and an enhanced intermediate-mass dileptons from charm meson decays. These results thus give a more quantitative justification of the arguments proposed in Ref. [4].

However, the results obtained in the present study are still incomplete as we have not included diagrams involving the exchange of heavier particles such as charm hadrons. The scattering cross sections between charm mesons and hadrons such as kaon, $\omega, \eta, \Delta$, and higher baryon resonances are not calculated either. Furthermore, we have not calculated the contribution due to diagrams shown in Fig. 4, where charm mesons scatter with nucleons via a $\Lambda_{c}$ exchange. There is a large uncertainty in their contributions as no empirical information on the coupling constant $g_{D N \Lambda_{c}}$ is available. The SU(4) symmetry gives

$$
g_{D N \Lambda_{c}}=g_{K N \Lambda}=\frac{3-2 \alpha_{D}}{\sqrt{3}} g_{\pi N N} \simeq g_{\pi N N}=13.5,
$$

where $\alpha_{D}=D /(D+F)$ with $D$ and $F$ being the coefficients for the $D$-type and $F$-type coupling, and $\alpha_{D} \simeq 0.64$ [18]. On the other hand, QCD sum rule studies suggest a smaller value $g_{D N \Lambda_{c}} \simeq 6.7 \pm 2.1[19]$. Because of this uncertainty in these two processes involving the $D N \Lambda_{c}$ coupling, we choose to leave them out in our study. In the future, when empirical information on this parameter is known from $D N$ scattering, then processes involving the $D N \Lambda_{c}$ coupling can be addressed.

We note that the estimates given above are based on a simple assumption on the time evolution of the dense hadronic system, which enables us to make an analytical estimate of the rescattering effects. As a result, we have neglected the transverse expansion of the hadronic system which would lead to a faster decrease of hadron densities than the linear dropping assumption in Eq. (5). We have also neglected the chemical equilibration processes which, e.g., may decrease the total number of rho mesons and increase that of pions as a function of time [17].

Moreover, we have used only the isospin averaged cross sections and also averaged the rescattering effects on $D$ and $D^{*}$ mesons. Without a full cascade calculation and fully treating the isospin, we do not know the final composition of charm mesons, e.g, the ratios $D^{*} / D$ and $D^{0} / D^{+}$. A naive expectation gives $D^{*} / D=3$, and consequently $D^{0} / D^{+} \simeq 3$ [20]. However, even for $p p$ collisions the relative weights of produced charm mesons are not well measured experimentally. We emphasize that the charm meson composition could have a sizable effect on the lepton and dilepton yields from charm decays, because $D^{+}$and $D^{0}$ have very different branching ratios for semileptonic decays $\left(17.2 \%\right.$ from $D^{+}$ and $6.7 \%$ from $D^{0}$ ).

In a hadronic cascade model, the time evolution and the chemical equilibration of the hadronic system can be simulated much better. Using cross sections with the full isospin information, and keeping track of the charm meson isospins during scatterings, the final charm meson composition can be determined. Therefore, further studies based a cascade code along these directions are much needed for a quantitative study of the rescattering effects on charm meson observables.

For heavy ion collisions at Relativistic Heavy Ion Collider (RHIC) energies, a dense partonic system is expected to be formed during the early stage of the collision. In addition to hadronic rescatterings of charm mesons, partonic rescattering effects on charm quarks also need to be included. Furthermore, radiative processes of charm quarks inside the QGP would further complicate the issue as they may cause energy loss [21] and soften the charm meson $m_{\perp}$ and pair invariant mass spectra [22]. Therefore, more studies are needed before one can make predictions for RHIC.

\section{ACKNOWLEDGMENTS}

We thank S. Vance, X.-N. Wang, and Pang Yang for helpful discussions. This work was supported in part by the National Science Foundation under Grant No. PHY9870038, the Welch Foundation under Grant No. A-1358, and the Texas Advanced Research Project No. FY97 010366-068.

\section{APPENDIX}

In this appendix, we derive the relation between the total squared momentum transfer to charm mesons due to hadronic scatterings and the increase of the inverse slope of charm meson $m_{\perp}$ spectra. Consider a charm meson at central rapidity with an initial transverse momentum $p_{\perp \mathrm{I}}$ along the $y$ axis. After a scattering which gives the charm meson a momentum $\vec{p}_{\mathrm{S}}$ in its rest frame, its final transverse momentum is given by

$$
p_{x \mathrm{~F}}=p_{x \mathrm{~S}}, \quad p_{y \mathrm{~F}}=\gamma_{\mathrm{I}}\left(p_{y \mathrm{~S}}+\beta_{\mathrm{I}} E_{\mathrm{S}}\right)
$$

where

$$
\beta_{\mathrm{I}}=\frac{p_{\perp \mathrm{I}}}{\sqrt{p_{\perp \mathrm{I}}^{2}+m^{2}}}, \quad \gamma_{\mathrm{I}}=\frac{1}{\sqrt{1-\beta_{\mathrm{I}}^{2}}} .
$$

Assuming that $\vec{p}_{\mathrm{S}}$ is isotropic in the charm meson rest frame, the average of the squared final transverse momentum of the charm meson is then related to that of the squared initial transverse momentum by

$$
\left\langle p_{\perp \mathrm{F}}^{2}\right\rangle=\left\langle p_{\perp \mathrm{I}}^{2}\right\rangle+\left(\frac{2}{3}+\frac{4\left\langle p_{\perp \mathrm{I}}^{2}\right\rangle}{3 m^{2}}\right)\left\langle p_{\mathrm{S}}^{2}\right\rangle
$$


where $\left\langle p_{\mathrm{S}}^{2}\right\rangle$ is the average of the squared total momentum transfer to the charm meson as given by Eq. (6). For an isotropic $\vec{p}_{\mathrm{S}}$ distribution, Eq. (A1) is actually true for a charm meson at any rapidity.

If we parametrize the $m_{\perp}$ spectrum of charm mesons as

$$
\frac{d N}{m_{\perp} d m_{\perp}} \propto e^{-m_{\perp} / T_{\text {eff }}}
$$

in terms of an inverse slope parameter $T_{\text {eff }}$, then

$$
\left\langle p_{\perp}^{2}\right\rangle=2 T_{\mathrm{eff}}^{2}\left(\frac{m}{T_{\mathrm{eff}}}+2+\frac{1}{m / T_{\mathrm{eff}}+1}\right) .
$$

As in the schematic study of Ref. [4], we characterize the scattering strength $\left\langle p_{\mathrm{S}}^{2}\right\rangle$ by an equivalent temperature parameter $T_{\mathrm{S}}$ via

$$
\begin{aligned}
\left\langle p_{\mathrm{S}}^{2}\right\rangle & =\frac{\int p^{2} e^{-E / T_{\mathrm{S}}} d^{3} p}{\int e^{-E / T_{\mathrm{S}}} d^{3} p}=T_{S}^{2} \frac{\int x^{4} \exp \left[-\sqrt{x^{2}+\left(m / T_{\mathrm{S}}\right)^{2}}\right] d x}{\int x^{2} \exp \left[-\sqrt{x^{2}+\left(m / T_{\mathrm{S}}\right)^{2}}\right] d x} \\
& =3 T_{\mathrm{S}}^{2}\left[\frac{m}{T_{\mathrm{S}}}+\frac{5}{2}+\mathcal{O}\left(\frac{1}{m / T_{\mathrm{S}}}\right)\right] .
\end{aligned}
$$

Both $T_{\mathrm{S}}$ and $T_{\text {eff }}$ are expected to be small compared with the charm meson mass $(m \simeq 1.87 \mathrm{GeV})$. Keeping only the leading term in Eqs. (A2) and (A3) then gives $\left\langle p_{\perp}^{2}\right\rangle$ $=2 m T_{\text {eff }}$ and $\left\langle p_{\mathrm{S}}^{2}\right\rangle=3 m T_{\mathrm{S}}$ in the nonrelativistic limit. Equation (A1) thus gives

$$
T_{\mathrm{eff}}^{\mathrm{F}} \simeq T_{\mathrm{eff}}^{\mathrm{I}}+T_{\mathrm{S}} .
$$

If we also keep the next-to-leading term in Eqs. (A2) and (A3), we then obtain

$$
\left\langle p_{\mathrm{S}}^{2}\right\rangle \simeq 3 m T_{\mathrm{S}}+\frac{15}{2} T_{\mathrm{S}}^{2}
$$

and

$$
\begin{aligned}
T_{\text {eff }}^{\mathrm{F}}+\frac{2 T_{\text {eff }}^{\mathrm{F}}}{m} \simeq & T_{\text {eff }}^{\mathrm{I}}+\frac{2 T_{\text {eff }}^{\mathrm{I}}}{m}+T_{S}\left(1+\frac{5 T_{\mathrm{S}}}{2 m}\right) \\
& \times\left[1+\frac{4 T_{\text {eff }}^{\mathrm{I}}}{m}\left(1+\frac{2 T_{\text {eff }}^{\mathrm{I}}}{m}\right)\right] .
\end{aligned}
$$

For central $\mathrm{Pb}+\mathrm{Pb}$ collisions at SPS energies, the relations given by Eqs. (A4) and (A6) are shown in Fig. 3 together with the results obtained from the Monte Carlo simulations in the schematic study of Ref. [4]. These relations agree qualitatively with that from the simulations. However, they differ quantitatively, because rapidity changes due to rescatterings are not taken into account in the present analytical estimates.
[1] M. Masera for the HELIOS-3 Collaboration, Nucl. Phys. A590, 93c (1995).

[2] E. Scomparin for the NA50 Collaboration, Nucl. Phys. A610, 331c (1996).

[3] G. Q. Li and C. Gale, Nucl. Phys. A638, 491c (1998).

[4] Z. Lin and X.-N. Wang, Phys. Lett. B 444, 245 (1998).

[5] B. L. Combridge, Nucl. Phys. B151, 429 (1979).

[6] P. Nason, S. Dawson, and R. K. Ellis, Nucl. Phys. B303, 607 (1988); B327, 49 (1989); W. Beenakker, H. Kuijf, W. L. van Neerven, and J. Smith, Phys. Rev. D 40, 54 (1989).

[7] S. K. Bose and W. D. McGlinn, Phys. Rev. D 14, 3167 (1976).

[8] Particle Data Group, C. Caso et al., Eur. Phys. J. C 3, 1 (1998).

[9] P. Colangelo, F. De Fazio, and G. Nardulli, Phys. Lett. B 334, 175 (1994).

[10] E. J. Eichten, C. T. Hill, and C. Quigg, Phys. Rev. Lett. 71, 4116 (1993); Y.-B. Dai and H.-Y. Jin, Phys. Rev. D 52, 236 (1995)

[11] C. M. Ko and D. Seibert, Phys. Rev. C 49, 2198 (1994); G. E. Brown, C. M. Ko, Z. G. Wu, and L. H. Xia, Phys. Rev. C 43, 1881 (1991).

[12] S. G. Matinyan and B. Müller, Phys. Rev. C 58, 2994 (1998). Note that our coupling constant is a factor of 2 smaller due to the difference of the definitions.

[13] B. Holzenkamp, K. Holinde, and J. Speth, Nucl. Phys. A500, 485 (1989); G. Janssen, J. W. Durso, K. Holinde, B. C. Pearce, and J. Speth, Phys. Rev. Lett. 71, 1978 (1993).

[14] G. Janssen, K. Holinde, and J. Speth, Phys. Rev. C 54, 2218 (1996).

[15] G. Q. Li and C. M. Ko, Nucl. Phys. A594, 439 (1995); W. Peters, U. Mosel, and A. Engel, Z. Phys. A 353, 333 (1996).

[16] R. Baier, M. Dirks, and K. Redlich, Phys. Rev. D 55, 4344 (1997).

[17] G. Q. Li, C. M. Ko, G. E. Brown, and H. Sorge, Nucl. Phys. A611, 539 (1996).

[18] R. A. Adelseck and B. Saghai, Phys. Rev. C 42, 108 (1990).

[19] F. S. Navarra and M. Nielsen, Phys. Lett. B 443, 285 (1998).

[20] S. Frixione, M. L. Mangano, P. Nason, and G. Ridolfi, hep-ph/9702287.

[21] R. Baier, Yu. L. Dokshitser, A. H. Mueller, S. Peigné, and D. Schiff, Nucl. Phys. B478, 577 (1996); B483, 291 (1997); R. Baier, Yu. L. Dokshitzer, A. H. Mueller, and D. Schiff, Phys. Rev. C 58, 1706 (1998).

[22] Z. Lin, R. Vogt, and X.-N. Wang, Phys. Rev. C 57, 899 (1998). 